\title{
A quality improvement project to improve the documentation of resuscitation and ceiling of care decisions in elderly patients at a large district general hospital
}

\author{
Authors: M Asim T Khan, Mohammad V Hamza, Huiyi E Law, Raidah Haider, Donna Walker and Sarath Wicks
}

\begin{abstract}
Aims
All elderly patients seen on post-take ward rounds should be assessed by the Gold Standards Framework (GSF), with resuscitation status and ceiling of care discussed and documented accordingly in the medical notes and discharge letters.

\section{Methods}

We opportunistically reviewed the medical notes and discharge letters of all patients aged over 75 years immediately prior to discharge. We conducted three cycles of data collection, each lasting 1 week in duration, from November 2017 to January 2018. Data was recorded into an anonymised Excel template. We determined a pre-intervention baseline in cycle one, with a view to compare the trend after implementing two interventions separately in the next cycles. In cycle two, we delivered a presentation at the quarterly geriatrics meeting. In cycle three, we emailed recommendations to all doctors based at the trust. We determined the outcome of each intervention.
\end{abstract}

\section{Results}

In the pre-intervention sample ( $n=35)$, only $51 \%$ patients had GSF documented. In cycle two $(n=45)$ this was $49 \%$ and in cycle three $(n=22)$ this fell to $32 \%$. In cycle one, $65 \%(13 / 20)$ of patients deemed 'not for resuscitation' (NFR) had no documented escalation status in the notes. In cycle two this was $46 \%(12 / 26)$ of NFR patients, and in cycle three this fell further to $14 \%(1 / 7)$. NFR status in cycle one was not communicated in $70 \%(14 / 20)$ of patients' discharge letters. In cycle two this fell to $35 \%(9 / 26)$ and rose to $100 \%(7 / 7)$ in cycle three. Seven patients met the GSF criteria in cycle one, 13 patients in cycle two, and four patients in cycle three. Regarding GSF patients in cycle one, $28 \%$ had no documented resuscitation discussion and $57 \%$ had no documented ceiling of care. In cycle two all GSF patients had a documented resuscitation discussion and only $25 \%$ had no documented ceiling of care. In cycle three, $75 \%$

Authors: Barking, Havering and Redbridge University Hospital Trust, London, UK had no resuscitation discussion documented and $25 \%$ had no documented ceiling of care.

\section{Conclusion}

The study demonstrates an overall improvement in documentation, particularly with the second cycle intervention. However, there is a relapse in standards in cycle three. This implies that the direct delivery of teaching sessions is far more effective than blanket emails. It also suggests that interventions need repeated administration to achieve the desired adequacy in documentation. The subpar documentation in cycle three may reflect the increased winter pressures at this point in the study and may also be confounded by the low discharge rates reducing sample size.

\section{Conflict of interest statement}

The authors collectively note reviewing a very small number of patients whose documentation had been completed by themselves, but the effect was not considered large enough to influence the results. 